\title{
Comparison of the Effects of lodixanol and lopamidol on Urine Flow, Urine Viscosity, and Glomerular Filtration in Rats
}

\author{
Keywords: lodinated contrast media; Viscosity; Glomerular \\ filtration rate; Rat; Hydration; Acute kidney injury; Contrast induced \\ nephropathy
}

\begin{abstract}
Dehydration is a major risk for contrast media (CM) induced acute kidney injury, yet prospective studies in human patients without ample hydration are not feasible. The freely drinking rat can serve as a model for hydropenic humans.

We compared the effects of two $C M$, iso-osmolar iodixanol and lowosmolar iopamidol, on urine flow, urine viscosity and glomerular filtration rate (GFR) in non-hydrated rats, and studied the impact of hydration by saline. In four groups of rats, either iodixanol $320 \mathrm{mg}$ iodine $/ \mathrm{mL}$ or iopamidol $370 \mathrm{mg}$ iodine $/ \mathrm{mL}$ was injected as $1.5 \mathrm{~mL}$ bolus into the thoracic aorta. Two groups had access to drinking water only and two groups received additional saline infusion $(4 \mathrm{~mL} /$ hour per $\mathrm{kg}$ ) starting $60 \mathrm{~min}$ before $C M$ injection. Urine was collected (10 min sampling periods), urine viscosity measured, and GFR determined by creatinine clearance. In nonprehydrated rats, iodixanol led to a massive increase in urine viscosity and a transient $50 \%$ drop in GFR. lopamidol had a much stronger diuretic effect than iodixanol, urine viscosity increased much less and GFR was unaffected. Saline infusion blunted the viscosity rise and transient decline in GFR caused by iodixanol. It is concluded that the choice of $C M$ and ample hydration are important elements in the prevention of $C M$ induced kidney injury. Low-osmolar iopamidol has a better renal safety profile than iso-osmolar iodixanol, at least in non-hydrated subjects. Hydration by saline counteracts the renal tubular concentration of $C M$ thereby alleviating the increase in urine viscosity and the decline in GFR.
\end{abstract}

\section{Introduction}

Although today's iodinated X-ray contrast media (CM) are well tolerated in general, there is still the risk of acute kidney injury following the intravascular administration of CM. This is especially true for procedures such as percutaneous cardiac interventions that often require large amounts of $\mathrm{CM}$ and entail intra-arterial $\mathrm{CM}$ administration [1-4]. Besides conditions such as pre-existing renal impairment and diabetes mellitus, volume depletion (dehydration) is a major risk factor for contrast media induced nephropathy (CIN) $[1,2,4,5]$.

$\mathrm{CM}$ of the pioneer generation, later on named high-osmolar CM (HOCM), are no longer widely used for intravascular administration, because these compounds showed a higher incidence of CIN than low-osmolar CM (LOCM) and iso-osmolar CM (IOCM) [1,2,6,7]. Whereas the osmolality of LOCM is much lower than that of HOCM but still higher than that of blood, IOCM have the same osmolality as blood. Today, we can choose between iodixanol, the only IOCM presently approved for intravascular administration, and an array of LOCM, such as the widely used iopamidol. Current guidelines leave

\section{Journal of}

Urology \& Nephrology

\author{
Mechthild Ladwig ${ }^{1,2 *}$, Kathleen Cantow ${ }^{1}$, Bert \\ Flemming $^{1}$, Pontus B. Persson ${ }^{1}$ and Erdmann \\ Seeliger ${ }^{1}$ \\ ${ }^{1}$ Institut für vegetative Physiologie and Cardiovascular Research \\ Center, Charité-Universitätsmedizin, Berlin, Germany \\ ${ }^{2}$ Institut für Tierschutz, Tierverhalten und Versuchstierkunde, Freie \\ Universität Berlin , Germany
}

\section{Address for Correspondence}

Mechthild Ladwig, Institut für vegetative Physiologie and Cardiovascular Research Center Charité-Universitätsmedizin, Berlin Hessische Str. 3-4 10115 Berlin, Germany, Tel: 004930450528196, Fax: 004930450528972 ; E-mail: mechthild.ladwig@charite.de

Submission: 30 June 2015

Accepted: 31 July 2015

Published: 04 August 2015

Copyright: ( 2015 Ladwig M, et al. This is an open access article distributed under the Creative Commons Attribution License, which permits unrestricted use, distribution, and reproduction in any medium, provided the original work is properly cited.

Reviewed \& Approved by: Dr. Ahmed El-Zawahry, Assistant Professor of Surgery, Southern Illinois University, USA this choice to the physician $[1,6-8]$, because the results of prospective clinical head-to-head comparisons concerning the renal safety of iodixanol versus LOCM have been contradictory [2,9-12]. One major reason behind this lack of consistent results is that the vast majority of clinical studies have been relying on serum creatinine concentration (SCrea) as the sole end point $[2,9,11,12]$. Unfortunately, SCrea is a notoriously poor surrogate marker for glomerular filtration rate (GFR): SCrea is quite insensitive in patients with normal pre-existing GFR and conceals quick and transient changes in GFR such as an immediate drop upon CM administration $[2,13,14]$.

A second reason behind the equivocal results of prospective clinical studies relies on the fact that- compelled by the knowledge that dehydration poses a grave risk for CIN-virtually all study protocols prescribed ample prehydration of the patients. This is in contrast to every-day clinical practice, in which many patients are not sufficiently hydrated $[15,16]$. Two register studies that mirror every-day practice and, thus, certainly included patients who were not well hydrated, point at a higher incidence of CIN following the IOCM, iodixanol, than LOCM $[17,18]$. A mechanistic explanation for this finding is 
Citation: Ladwig M, Cantow K, Flemming B, Persson PB, Seeliger E. Comparison of the Effects of lodixanol and lopamidol on Urine Flow, Urine Viscosity, and Glomerular Filtration in Rats. J Urol Nephrol. 2015;2(1): 7.

that, in less hydrated subjects; water resorption from renal tubular fluid is enhanced, leading to an increase in the tubular concentration of CM. This will result in a proportional increase in tubular fluid osmolality and an over proportional increase in its viscosity. Both high tubular fluid osmolality and viscosity have been implicated to promote CIN $[2,17,19,20]$. IOCM have a higher fluid viscosity than LOCM [21,22], which, in patients who are not well hydrated, may result in a higher incidence of CIN.

Because prospective studies in humans without proper prehydration are, of course, unfeasible for ethical reasons, the ultimate appraisal of the renal safety of IOCM versus LOCM in this condition must be gained by animal experiments. Euhydrated rats which have free access to water concentrate their urine to $900-1200 \mathrm{mosmol} / \mathrm{kg}$ $\mathrm{H}_{2} \mathrm{O}$, values that compare well with urine osmolality measured for hydropenic human beings [23-25]. Studies in rats also facilitate direct measurements of GFR with high temporal solution. Finally, with the high level of standardisation enabled by studies in healthy rats, the issues of heterogeneities among patients in clinical studies, and, even more pronounced, in meta-analyses derived from clinical studies, can be circumvented.

For these reasons we performed a head-to-head comparison of the IOCM, iodixanol, and the LOCM, iopamidol, in anaesthetized rats. We chose to inject the $\mathrm{CM}$ into the thoracic aorta to emulate the clinical setting of percutaneous cardiac interventions. We hypothesized that iodixanol results in higher urine viscosity and, thus, in larger decrease in GFR as compared to iopamidol in rats that are not hydrated by infusion. In order to study the effects of pre-hydration, we performed a second set of experiments in rats that received a continous i.v. infusion of isotonic saline. We hypothesized that saline alleviates the drop in GFR and lessens the rise in urine viscosity.

\section{Material and Methods}

This study was approved by Berlin's Legal Authorities and performed in accordance with the German Animal Welfare Act. All applicable institutional and national guidelines for the care and use of animals were followed.

For the experiments 3-4 months old male Wistar rats (body mass 350-480g; Harlan, Roßdorf, Germany) were used. A standard diet was fed and the rats had free access to drinking water. As enrichment measures they were housed in groups in type IV cages and nesting material and plastic tubes were offered.

\section{Experimental protocols and procedures}

The study comprised experiments on a total of 36 rats, which were allocated into four treatment groups. The protocols differed with respect to the hydration regimen and the administered $\mathrm{CM}$. All rats had free access to drinking water until anaesthesia, yet the rats in two groups received an additional continuous infusion of isotonic $\mathrm{NaCl}$ solution (saline) at $4 \mathrm{~mL}$ per hour per $\mathrm{kg}$ of body mass (BM). This infusion was started 60 min before administration of $\mathrm{CM}$ and was continued throughout the observation time (100 min post CM). The rats in two groups were injected with iodixanol $320 \mathrm{mg}$ iodine per $\mathrm{mL}$ (Visipaque ${ }^{\circledast}$ 320; GE Healthcare Buchler, Braunschweig, Germany) whereas the rats in the other two groups received iopamidol $370 \mathrm{mg}$ iodine per $\mathrm{mL}$ (Iopamiron ${ }^{\circ}$ 370; Bayer, Osaka, Japan). According to manufacturers' specifications the osmolality of iodixanol 320 solution is $290 \mathrm{mosmol} / \mathrm{kg} \mathrm{H}_{2} \mathrm{O}$, that of iopamidol 370 solution is 796 mosmol/ $\mathrm{kg} \mathrm{H} \mathrm{H}_{2} \mathrm{O}$, their viscosities (at $37{ }^{\circ} \mathrm{C}$ ) are $11.4 \mathrm{mPa} \mathrm{s}$ and 9.1 $\mathrm{mPa}$ s, respectively. Note that rats receiving $1.5 \mathrm{~mL}$ of iopamidol 370 are exposed to a greater amount of iodine than rats receiving $1.5 \mathrm{~mL}$ of iodixanol 320. CM were pre-warmed to $37^{\circ} \mathrm{C}$ and administered into the thoracic aorta as a bolus injection within a timeframe of 20 $\mathrm{s}$. The four groups were named 1) iodixanol ( $\mathrm{n}=9$ rats), 2) iopamidol $(\mathrm{n}=10), 3)$ iodixanol+saline $(\mathrm{n}=7)$, and 4$)$ iopamidol+saline $(\mathrm{n}=10)$.

The experiments started at 8:00 a.m. Rats were anaesthetized by intraperitoneal injection of $20 \%$ urethane solution $(6 \mathrm{~mL} / \mathrm{kg} \mathrm{BM})$ and fixed on a temperature controlled table. A tracheal cannula was inserted to ensure sufficient spontaneous breathing. Catheters were inserted into blood vessels - one into the left common carotid artery with its tip towards the aorta for $\mathrm{CM}$ administration, another one into the left jugular vein for infusion, and a third one into the femoral artery for taking blood samples and monitoring arterial blood pressure. Urine was collected via a urinary bladder catheter inserted by opening the abdominal cavity.

Urine sampling was done in consecutive 10 min sampling periods. Blood samples were taken before $\mathrm{CM}$ administration and at the end of the observation period.

\section{Measurements}

Urine volume was measured gravimetrically. Osmolality was measured by freezing point depression (Osmomat 030; Gonotec, Berlin, Germany). Glomerular filtration rate was measured by clearance of creatinine. To this end, creatinine concentration in serum and urine samples was measured (Creatinine Analyzer II; Beckmann Instruments, Galway, Ireland; SCrea between both samples estimated by linear interpolation), and clearance calculated by standard formula. Viscosity of urine was measured by a microviscometer developed in our laboratory that enables measurements from minute samples [26]. Viscosity of urine samples was measured at $37^{\circ} \mathrm{C}$ sample temperature.

Although normal urine flow is very sparse in rats which are not hydrated by infusions, we could secure enough urine for the viscometry $(7 \mu \mathrm{L})$. However, it was not possible to obtain enough urine for calculating flow rates and creatinine clearances before giving $\mathrm{CM}$ in the rats that did not receive saline infusions. After $\mathrm{CM}$ injection, urine samples were large enough in all rats for all analyses, because $\mathrm{CM}$ elicits osmodiuresis.

\section{Statistical analysis}

Statistical comparisons among the protocols (unpaired data) were made with the Kruskal-Wallis test (one-way analysis of variance on ranks) followed by the Dunn procedure for multiple non-parametric comparisons. Comparisons within a given protocol (paired data) were performed with the Friedmann test (repeated-measurement analysis of variance on ranks). Statistical software (NCSS; Hintze, Kaysville Utah) was used, with a significance level of $\mathrm{P}<0.05$. Data are presented as means \pm SEM.

\section{Results}

In the rats that had access to drinking water only, pre-CM urine flow was as miniscule and urine osmolality was as high as expected 
Citation: Ladwig M, Cantow K, Flemming B, Persson PB, Seeliger E. Comparison of the Effects of Iodixanol and Iopamidol on Urine Flow, Urine Viscosity, and Glomerular Filtration in Rats. J Urol Nephrol. 2015;2(1): 7.

(1016 $\pm 94 \mathrm{mosmol} / \mathrm{kg} \mathrm{H}_{2} \mathrm{O}$; it did not differ significantly between the iopamidol and the iodixanol group). CM administration immediately increased urine flow rate, and this effect persisted throughout the 100 min observation period (Figure 1A). The diuretic effect of iopamidol was significantly stronger than that of iodixanol: the cumulative urine volume for the $100 \mathrm{~min}$ post $\mathrm{CM}$ administration was $2.24 \pm 0.08 \mathrm{~mL}$ in the iopamidol group and $1.35 \pm 0.10 \mathrm{~mL}$ in the iodixanol group (Figure 1C).

As saline had been infused for $60 \mathrm{~min}$ in the iopamidol+saline and iodixanol+saline groups, pre-CM urine flow rate was higher
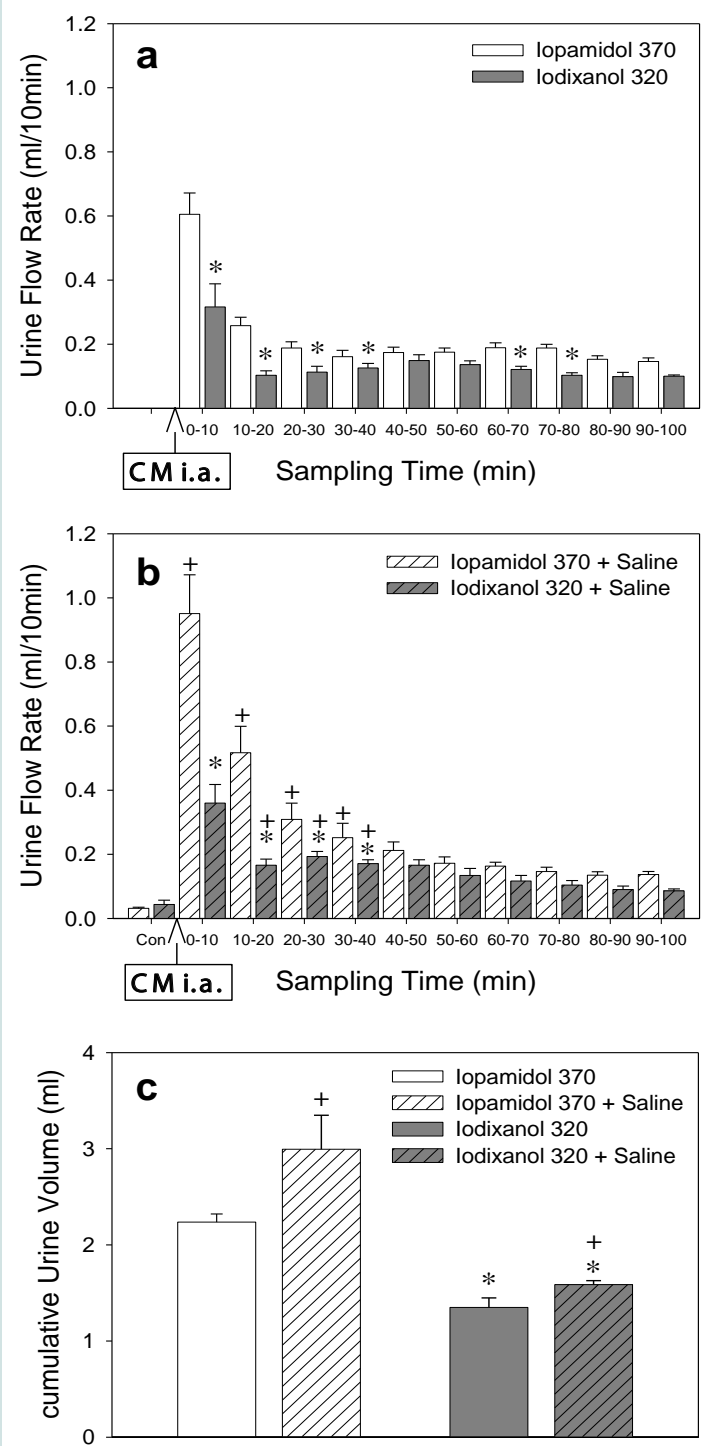

Figure 1: Urine flow rates (mean \pm SEM) before (Con) and following $\mathrm{CM}$ bolus injection into the thoracic aorta (i.a.). (A) Results of rats that had access to drinking water only, $(B)$ results of rats that additionally received saline infusion $(4 \mathrm{~mL} / \mathrm{h}$ per $\mathrm{kg} \mathrm{BM}$ ) initiated 60 min before $\mathrm{CM}$, (C) cumulative urine volumes for the 100 min following $\mathrm{CM}$ for all four groups. Note that urine flow pre-CM in rats not pre-hydrated by saline was too sparse to obtain flow rates. In al sample periods after $\mathrm{CM}$, urine flow was higher than during the respective control period. Significant differences between iodixanol and iopamidol are depicted by *, those between the hydration states by $+(p<0.05)$.
(Figure 1B) and pre-CM urine osmolality was significantly lower (551 $\pm 87 \mathrm{mosmol} / \mathrm{kg} \mathrm{H}_{2} \mathrm{O}$; no significant difference between the iopamidol+saline and the iodixanol+saline group) than in the iopamidol and iodixanol groups. The continued saline infusion also increased the diuresis following administration of both CM (Figure $1 \mathrm{~B})$, yet the cumulative urine volume for the $100 \mathrm{~min}$ post-CM period was still significantly smaller for the iodixanol+saline group $(1.59 \pm 0.04 \mathrm{~mL})$ than that of the iopamidol+saline $(3.00 \pm 0.35 \mathrm{~mL})$ and even smaller than that of the iopamidol group (Figure 1C).

Pre-CM urine viscosity values did not differ between the iopamidol group and the iodixanol group (Figure 2A). Both iodixanol and iopamidol resulted in significant increases in urine viscosity, yet values were much higher in the iodixanol (peak value $32.2 \pm 7.1 \mathrm{~mm}^{2} / \mathrm{s}$ ) than in the iopamidol group (peak value $2.2 \pm 0.3 \mathrm{~mm}^{2} / \mathrm{s}$ ). Infusion of isotonic saline for $60 \mathrm{~min}$ did not alter pre-CM values of urine viscosity (Figure $2 \mathrm{~B}$ ). The continued saline infusion significantly decreased urine viscosities following iodixanol (iodixanol+saline group peak value $16.2 \pm 2.6 \mathrm{~mm}^{2} / \mathrm{s}$ ), whereas it did not significantly reduce the already much lower urine viscosity following iopamidol (iopamidol+saline group peak value $1.8 \pm 0.1 \mathrm{~mm}^{2} / \mathrm{s}$ ).

Glomerular filtration rate as measured by creatinine clearance decreased markedly following iodixanol (lowest value $5.3 \pm 0.9 \mathrm{~mL} /$ $10 \mathrm{~min}$ ); it became restored towards control level beginning $40 \mathrm{~min}$ post iodixanol (Figure 3A). Following iopamidol, GFR did not drop significantly (lowest value $11.7 \pm 1.7 \mathrm{~mL} / 10 \mathrm{~min}$ ). Please note that the large increase in creatinine clearance in the first 10 min sampling period after CM bolus does not represent an increase in GFR but relies on the well-known dead space effect: Due to CM induced massive diuresis, tubular fluid of very high creatinine concentration is flushed out of the urinary tract [26,27]. Saline infusion did not prevent the iodixanol-induced decrease in GFR completely (iodixanol+saline group lowest value $7.4 \pm 1.2 \mathrm{~mL} / 10 \mathrm{~min}$ ) but the drop was shortlasting: GFR was restored towards control values beginning about 20 min post-CM already.

\section{Discussion}

The present head-to-head comparison indicates that intra-arterial injection of iodixanol, but not iopamidol, leads to a vast increase in the renal tubular fluid viscosity (Figure 2) and to a substantial transient decrease in GFR (Figure 3) in healthy rats. Ample hydration with saline mitigates the increase in urine viscosity and diminishes the duration of GFR decline observed after iodixanol exposure. The results of this study supplement the literature on pathophysiological mechanisms causative for CIN and provide a lucid explanation for the beneficial effect of hydration.

In recent years it became possible, largely thanks to pre-clinical studies, to form a rather comprehensive view on the pathophysiology behind CIN [2,28-31]. CIN comes about by concerted action of cytotoxic effects of CM, auto-and paracrine factors which impinge on (intra) renal blood supply and oxygenation, and alterations of rheological properties that affect tubular fluid flow and renal haemodynamics. Yet the contributions of the individual factors vary with the physicochemical properties of the $\mathrm{CM}$ and the hydration status.

Medullary hypoxia is a key element in CIN pathophysiology 
Citation: Ladwig M, Cantow K, Flemming B, Persson PB, Seeliger E. Comparison of the Effects of lodixanol and lopamidol on Urine Flow, Urine Viscosity, and Glomerular Filtration in Rats. J Urol Nephrol. 2015;2(1): 7.
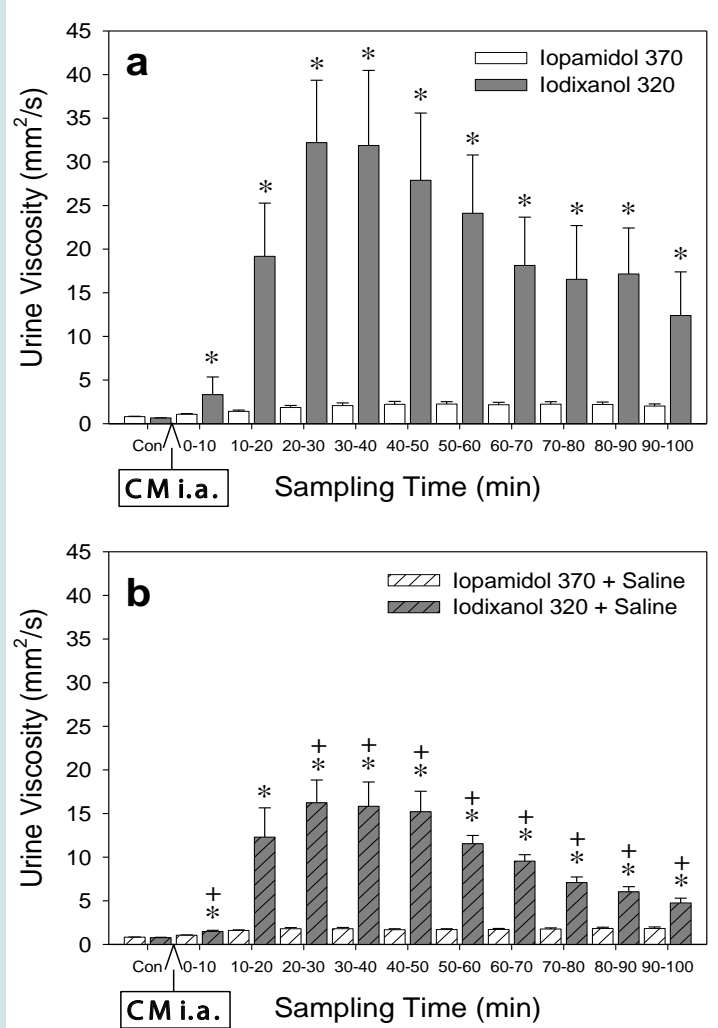

Figure 2: Viscosity of urine samples before and following $\mathrm{CM}$ administration (A) of rats that had access to drinking water only, (B) of rats that additionally received saline infusion. For groups and statistics see Legend to Fig. 1. In all urine samples after $\mathrm{CM}$, viscosity was significantly higher than in the respective control sample.

[2,20,28,29,32-35]. Hypoxia is part of a vicious circle that entails cellular damage, oxidative stress and vasoconstriction [2,28,29]. All presently used CM share cytotoxic effects that affect vascular endothelial and tubular epithelial cells $[2,36,37]$, which can lower the bioavailability of vasodilatory nitric oxide [36,37]. Pre-glomerular vasoconstriction is one reason behind CM-induced reduction in GFR [38], such as observed here upon iodixanol injection (Figure 3) [29]. Vasoconstriction of post-glomerular vessels including the descending vasa recta (DVR) will result in medullary hypoxia, and, thus, promote the vicious circle $[29,39]$.

That the viscosity of CM plays a role in CIN pathophysiology has been suggested in the 1990ies already [19]. The viscosities of all CM are markedly higher than those of blood plasma [21,22]. In the renal tubules, CM becomes enriched, as water is reabsorbed but CM is not. Because the relationship between concentration and viscosity is exponential, tubular fluid viscosity increases exponentially along the passage through the tubules. As recently reviewed [29], this has a number of deleterious effects. First, increased tubular fluid viscosity hinders glomerular filtration, as corroborated here: the massive increase in urine viscosity upon iodixanol is accompanied by a marked decrease in GFR (Figures $2 \mathrm{~A}$ and $3 \mathrm{~A}$ ). Second, the higher the tubular fluid viscosity the slower the CM is excreted (Figure 1A). This prolongs the contact time of cytotoxic CM with the tubular epithelial cells thereby aggravating the cell damage and the ensuing vasoconstrictive effects [22,29]. Third, viscosity-induced increase in tubular pressure compresses intrarenal vessels such as the DVR, thereby reducing medullary blood perfusion $[20,29,40]$. Finally, as blood flows through the hypertonic environment of the medulla, plasma water leaves the vessels towards the interstitium. This will enrich CM within the DVR, thus increasing blood viscosity, thereby further compromising medullary perfusion $[20,29]$.

The large difference observed between urine viscosities following iodixanol versus iopamidol (Figure $2 \mathrm{~A}$ ) cannot be explained by the difference of viscosities of the iodixanol 320 versus the iopamidol 370 solution alone ( 10 versus $\left.\sim 7 \mathrm{~mm}^{2} / \mathrm{s}\right)$. Here, the difference in CM osmolalities plays a major role (290 versus $796 \mathrm{mosmol} / \mathrm{kg} \mathrm{H}_{2} \mathrm{O}$ ) $[29,32,41]$. Non-reabsorbable CM diminish the osmotic gradient that drives tubular water resorption, thereby inducing osmodiuresis. The osmodiuretic effect of iopamidol is much stronger than that of iodixanol (Figure 1). By virtue of its higher osmolality, iopamidol is less enriched inside the tubules than iodixanol. Due to the exponential concentration-viscosity relationship, this results in a very modest increase in urine viscosity following iopamidol, but a vast increase in urine viscosity and, thus, a marked decrease in GFR following
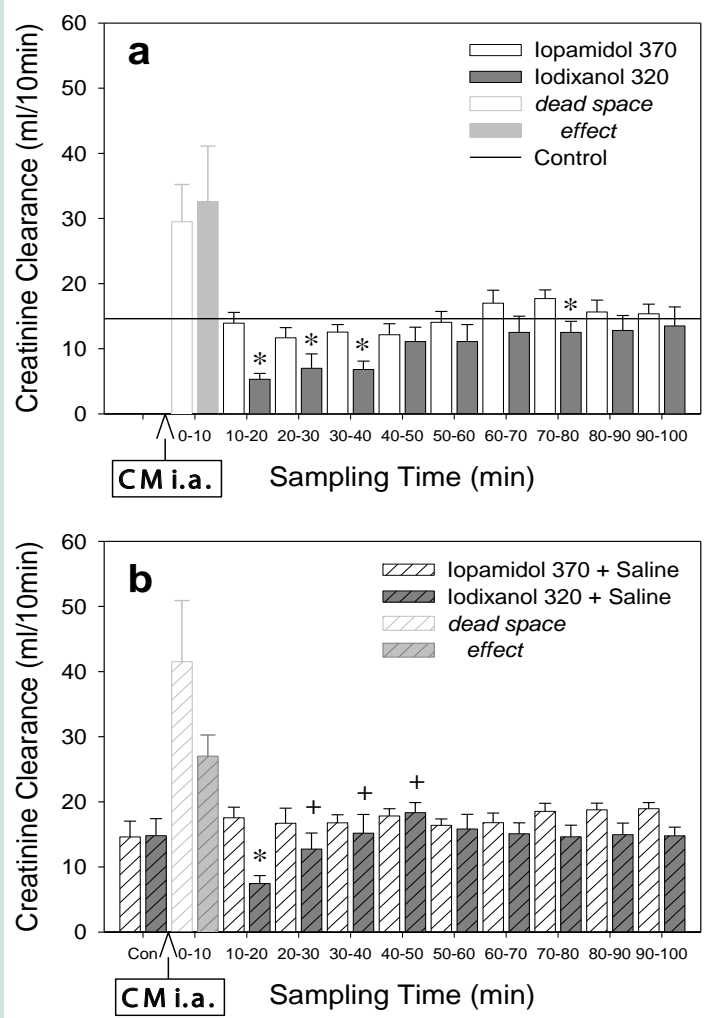

Figure 3: Creatinine clearance.

Creatinine clearances before and following $\mathrm{CM}(\mathrm{A})$ of rats that had access to drinking water only, (B) of rats that additionally received saline infusion. For groups and statistics see Legend to Fig. 1. Control values of creatinine clearance could only be obtained for rats receiving saline infusion (B); the mean value of these measurements is also depicted as Control in (A). Please note that the very high creatinine clearance values for the first sampling period following $\mathrm{CM}$ (0-10 min, depicted as grey shadows) do not represent actual increases in glomerular filtration rate, but rely on the dead-space effect (see Results). 
Citation: Ladwig M, Cantow K, Flemming B, Persson PB, Seeliger E. Comparison of the Effects of lodixanol and Iopamidol on Urine Flow, Urine Viscosity, and Glomerular Filtration in Rats. J Urol Nephrol. 2015;2(1): 7.

iodixanol (Figures $2 \mathrm{~A}$ and $3 \mathrm{~A}$ ). Thus, the modest osmolality of the LOCM, iopamidol, conveys a beneficial effect, at least in subjects that are not well hydrated.

The present results are in accord with previous comparisons between IOCM and various LOCM. As recently reviewed [29], these studies used several direct and indirect markers of kidney injury and consistently found that LOCM have a better renal safety profile than IOCM. Thus, urine viscosity was found higher in rats and dogs following IOCM than LOCM $[19,20,26,42]$; this was also corroborated in a small series of well hydrated patients [20]. Studies in rats and minipigs showed that the higher urine viscosity leads to a longer renal retention of IOCM than LOCM $[32,35,41,43]$, which is accompanied by higher levels of injury markers [32,41]. Also, intrarenal oxygenation was much more compromised following IOCM than LOCM as monitored by invasive probes and blood oxygenation leveldependent (BOLD) magnetic resonance [20,32,33,35].

Besides using the lowest CM dose possible, ample hydration (volume expansion) is the only unequivocally recommended measure for CIN prevention of today's guidelines [1,6-8]. The results of the present study provide a lucid explanation as regards the mechanism by which hydration (volume expansion) exerts its protective effect. The less a subject is hydrated the more activated are the mechanisms of volume preservation that govern tubular fluid resorption $[44,45]$. Thus, the degree to which CM are enriched en route through the tubules depends on the subject's hydration (volume status). With the volume expansion by saline, urine flow is increased (Figure 1) and, thus, the CM in the tubular fluid become less concentrated. Due to the exponential concentration-viscosity relation, this dilution results in a major decrease in urine viscosity following iodixanol (Figure 2). Consequently, the decline in GFR following iodixanol is alleviated (Figure 3).

A recent study in patients with congestive heart failure addressed legitimate concerns about excessive saline infusion [46]. Hence, left ventricular end-diastolic pressure was measured and the infusion rate adjusted accordingly. The higher the infusion rates were the lower the incidence of CIN was [46]. Another novel technique that significantly reduces the incidence of CIN combines forced diuresis with controlled hydration: Using a servocontrol device ("RenalGuard"), the rate of saline infusion is adjusted to match the urine output, thus providing volume expansion in the face of furosemide-forced diuresis $[47,48]$.

Our study has limitations. The euhydrated rat concentrates its urine to an extent which, in humans, is indicative of a hydropenic state (> $900 \mathrm{mosmol} / \mathrm{kg} \mathrm{H}_{2} \mathrm{O}$ ), however, the activity of mechanisms that control tubular fluid resorption will be higher in hydropenic humans. Yet, the euhydrated rat is probably the best available model for a situation that is not uncommon in daily clinical practice but ethically inadmissible to study in prospective clinical trials. By injecting $\mathrm{CM}$ into the thoracic aorta, we emulate percutaneous cardiac interventions. The CM dose used is in the lower range of the doses patients undergoing such interventions usually receive [49-52], as compared on a per body surface area basis as required for dose-dependent adverse effects between species by regulatory bodies [50]. However, during cardiac interventions the CM is usually given by consecutive smaller injections. In the present study, the whole amount of CM was given as a single bolus so that the first pass concentration of $\mathrm{CM}$ at the kidney is certainly higher than during most interventions in humans [53].

Taken together, the present study indicates that the choice of CM and ample hydration are important elements in the prevention of CIN. In clinical practice, as opposed to virtually all prospective trials on CIN including one head-to-head comparison between iopamidol and iodixanol [54], not every patient is sufficiently hydrated. Our study in the euhydrated rat model revealed that iopamidol, by virtue of its lower viscosity and moderately higher osmolality, has a better renal safety profile than iodixanol in this potentially harmful condition. Furthermore, the present results provide a mechanistic explanation for the renoprotective effect of hydration (volume expansion): Even a minor decrease in tubular water resorption resulting from hydration greatly decreases tubular fluid viscosity.

\section{References}

1. Ohno I, Hayashi H, Aonuma K, Horio M, Kashihara N, et al. (2013) Guidelines on the use of iodinated contrast media in patients with kidney disease 2012: digest version. JSN, JRS, and JCS Joint Working Group. Jpn J Radiol 31 : 546-584.

2. Seeliger E, Sendeski M, Rihal CS, Persson PB (2012) Contrast-induced kidney injury: mechanisms, risk factors, and prevention. Eur Heart J 33: $2007-$ 2015.

3. Solomon R, Dauerman HL (2010) Contrast-induced acute kidney injury. Circulation 122: 2451-2455

4. James MT, Samuel SM, Manning MA, Tonelli M, Ghali WA, et al. (2013) Contrast-induced acute kidney injury and risk of adverse clinical outcomes after coronary angiography: a systematic review and meta-analysis. Circ Cardiovasc Interv 6: 37-43.

5. Calvin AD, Misra S, Pflueger A (2010) Contrast-induced acute kidney injury and diabetic nephropathy. Nat Rev Nephrol 6: 679-688.

6. Stacul F, van der Molen AJ, Reimer P, Webb JA, Thomsen HS, et al. (2011) Contrast induced nephropathy: updated ESUR Contrast Media Safety Committee guidelines. Eur Radiol 21: 2527-2541.

7. American College of Radiology (2013) ACR Manual on Contrast Media Version 9.

8. Owen RJ, Hiremath S, Myers A, Fraser-Hill M, Barrett BJ (2014) Canadian Association of Radiologists consensus guidelines for the prevention of contrast-induced nephropathy: update 2012. Can Assoc Radiol J 65: 96-105.

9. Reed M, Meier P, Tamhane UU, Welch KB, Moscucci M, et al. (2009) The relative renal safety of iodixanol compared with low-osmolar contrast media: a meta-analysis of randomized controlled trials. JACC Cardiovasc Interv 2 : 645-654.

10. Biondi-Zoccai G, Lotrionte M, Thomsen HS, Romagnoli E, D'Ascenzo F, et al. (2014) Nephropathy after administration of iso-osmolar and low-osmolar contrast media: evidence from a network meta-analysis. Int J Cardiol 172 : 375-380.

11. Heinrich MC, Haberle L, Muller V, Bautz W, Uder M (2009) Nephrotoxicity of iso-osmolar iodixanol compared with nonionic low-osmolar contrast media: meta-analysis of randomized controlled trials. Radiology 250: 68-86.

12. From AM, Al Badarin FJ, McDonald FS, Bartholmai BJ, Cha SS, et al. (2010) lodixanol versus low-osmolar contrast media for prevention of contrast induced nephropathy: meta-analysis of randomized, controlled trials. Circ Cardiovasc Interv 3: 351-358.

13. McCullough PA, Shaw AD, Haase M, Bouchard J, Waikar SS, et al. (2013) Diagnosis of acute kidney injury using functional and injury biomarkers: workgroup statements from the tenth Acute Dialysis Quality Initiative Consensus Conference. Contrib Nephrol 182: 13-29.

14. Russo D, Minutolo R, Cianciaruso B, Memoli B, Conte G, et al. (1995) Early 
Citation: Ladwig M, Cantow K, Flemming B, Persson PB, Seeliger E. Comparison of the Effects of lodixanol and lopamidol on Urine Flow, Urine Viscosity, and Glomerular Filtration in Rats. J Urol Nephrol. 2015;2(1): 7.

effects of contrast media on renal hemodynamics and tubular function in chronic renal failure. J Am Soc Nephrol 6: 1451-1458.

15. Weisbord SD, Mor MK, Resnick AL, Hartwig KC, Sonel AF, et al. (2008) Prevention, incidence, and outcomes of contrast-induced acute kidney injury. Arch Intern Med 168: 1325-1332.

16. Fischereder M (2008) Use of intravenous sodium bicarbonate might increase the risk of contrast nephropathy. Nat Clin Pract Nephrol 4: 296-297.

17. Liss P, Persson PB, Hansell P, Lagerqvist B (2006) Renal failure in 57925 patients undergoing coronary procedures using iso-osmolar or low-osmolar contrast media. Kidney Int 70: 1811-1817.

18. Reed MC, Moscucci M, Smith DE, Share D, LaLonde T, et al. (2010) The relative renal safety of iodixanol and low-osmolar contrast media in patients undergoing percutaneous coronary intervention. Insights from Blue Cross Blue Shield of Michigan Cardiovascular Consortium (BMC2). J Invasive Cardiol 22: 467-472.

19. Ueda J, Nygren A, Sjoquist M, Jacobsson E, Ulfendahl HR, et al. (1998) lodine concentrations in the rat kidney measured by $\mathrm{X}$-ray microanalysis. Comparison of concentrations and viscosities in the proximal tubules and renal pelvis after intravenous injections of contrast media. Acta Radiol 39 90-95.

20. Seeliger E, Flemming B, Wronski T, Ladwig M, Arakelyan K, et al. (2007) Viscosity of contrast media perturbs renal hemodynamics. J Am Soc Nephro 18: $2912-2920$

21. Pannu N, Wiebe N, Tonelli M, Alberta Kidney Disease Network (2006) Prophylaxis strategies for contrast-induced nephropathy. JAMA 295: 27652779.

22. Jost G, Lengsfeld $P$, Lenhard DC, Pietsch H, Hutter J, et al. (2011) Viscosity of iodinated contrast agents during renal excretion. Eur J Radiol 80: 373-377.

23. Bozinovic F, Gallardo P (2006) The water economy of South American desert rodents: from integrative to molecular physiological ecology. Comp Biochem Physiol C Toxicol Pharmacol 142: 163-172.

24. Rapoport S, Brodsky WA, West CD (1949) Excretion of solutes and osmotic work of the resting kidney of hydropenic men. Am J Physiol 157: 357-362.

25. Manz F, Wentz A (2003) 24-h hydration status: parameters, epidemiology and recommendations. Eur J Clin Nutr 57 Suppl 2: S10-S18.

26. Seeliger E, Becker K, Ladwig M, Wronski T, Persson PB, et al. (2010) Up to 50 -fold increase in urine viscosity with iso-osmolar contrast media in the rat. Radiology 256: 406-414.

27. Badzynska B, Sadowski J, Dobrowolski L (1990) Atrial peptide natriuresis in the rat without genuine rise in filtration rate or wash-out of medullary electrolytes. J Physiol 427: 421-434.

28. Heyman SN, Rosen S, Khamaisi M, Idee JM, Rosenberger C (2010) Reactive oxygen species and the pathogenesis of radiocontrast-induced nephropathy Invest Radiol 45: 188-195.

29. Seeliger E, Lenhard DC, Persson PB (2014) Contrast media viscosity versus osmolality in kidney injury: lessons from animal studies. Biomed Res Int 2014 358136

30. Bucher AM, De Cecco CN, Schoepf UJ, Meinel FG, Krazinski AW, et al (2014) Is contrast medium osmolality a causal factor for contrast-induced nephropathy? Biomed Res Int 2014: 931413.

31. Aurelio A, Durante A (2014) Contrast-induced nephropathy in percutaneous coronary interventions: pathogenesis, risk factors, outcome, prevention and treatment. Cardiology 128: 62-72.

32. Lenhard DC, Frisk AL, Lengsfeld P, Pietsch H, Jost G (2013) The effect of iodinated contrast agent properties on renal kinetics and oxygenation. Invest Radiol 48: 175-182.

33. Li LP, Franklin T, Du H, Papadopoulou-Rosenzweig M, Carbray J, et al. (2012) Intrarenal oxygenation by blood oxygenation level-dependent MRI in contrast nephropathy model: effect of the viscosity and dose. J Magn Reson Imaging 36: 1162-1167.
34. Sekiguchi H, Ajiro Y, Uchida Y, Ishida I, Otsuki H, et al. (2013) Oxygen preconditioning prevents contrast-induced nephropathy (OPtion CIN Study). J Am Coll Cardiol 62: 162-163.

35. Wang YC, Tang A, Chang D, Zhang SJ, Ju S (2014) Significant perturbation in renal functional magnetic resonance imaging parameters and contrast retention for iodixanol compared with iopromide: an experimental study using blood-oxygen-level-dependent/diffusion-weighted magnetic resonance imaging and computed tomography in rats. Invest Radiol 49: 699-706.

36. Sendeski MM, Bondke PA, Liu ZZ, Busch JF, Weikert S, et al. (2012) lodinated contrast media cause endothelial damage leading to vasoconstriction of human and rat vasa recta. Am J Physiol Renal Physiol 303: F1592-F1598.

37. Liu ZZ, Schmerbach K, Lu Y, Perlewitz A, Nikitina T, et al. (2014) Iodinated contrast media cause direct tubular cell damage, leading to oxidative stress, low nitric oxide, and impairment of tubuloglomerular feedback. Am J Physio Renal Physiol 306: F864-F872.

38. Liu ZZ, Viegas VU, Perlewitz A, Lai EY, Persson PB, et al. (2012) Iodinated contrast media differentially affect afferent and efferent arteriolar tone and reactivity in mice: a possible explanation for reduced glomerular filtration rate. Radiology 265: 762-771.

39. Sendeski M, Patzak A, Pallone TL, Cao C, Persson AE, et al. (2009) lodixanol, constriction of medullary descending vasa recta, and risk for contrast medium-induced nephropathy. Radiology 251: 697-704.

40. Arakelyan K, Cantow K, Hentschel J, Flemming B, Pohlmann A, et al. (2013) Early effects of an x-ray contrast medium on renal T(2) $/ / T(2) \mathrm{MRI}$ as compared to short-term hyperoxia, hypoxia and aortic occlusion in rats. Acta Physiol (Oxf) 208: 202-213.

41. Lenhard DC, Pietsch H, Sieber MA, Ernst R, Lengsfeld P, et al. (2012) The osmolality of nonionic, iodinated contrast agents as an important factor for renal safety. Invest Radiol 47: 503-510.

42. Lancelot E, Idee JM, Lacledere C, Santus R, Corot C (2002) Effects of two dimeric iodinated contrast media on renal medullary blood perfusion and oxygenation in dogs. Invest Radiol 37: 368-375.

43. Jost G, Pietsch H, Lengsfeld P, Hutter J, Sieber MA (2010) The impact of the viscosity and osmolality of iodine contrast agents on renal elimination. Invest Radiol 45: 255-261

44. Reinhardt HW, Seeliger E (2000) Toward an integrative concept of control of total body sodium. News Physiol Sci 15: 319-325.

45. Seeliger E, Lunenburg T, Ladwig M, Reinhardt HW (2010) Role of the reninangiotensin-aldosterone system for control of arterial blood pressure following moderate deficit in total body sodium: balance studies in freely moving dogs. Clin Exp Pharmacol Physiol 37: e43-e51.

46. Brar SS, Aharonian V, Mansukhani P, Moore N, Shen AY, et al. (2014) Haemodynamic-guided fluid administration for the prevention of contrastinduced acute kidney injury: the POSEIDON randomised controlled trial. Lancet 383: 1814-1823.

47. Briguori C, Visconti G, Focaccio A, Airoldi F, Valgimigli M, et al. (2011) Renal Insufficiency After Contrast Media Administration Trial II (REMEDIAL II): RenalGuard System in high-risk patients for contrast-induced acute kidney injury. Circulation 124: 1260-1269.

48. Solomon R (2014) Forced diuresis with the RenalGuard system: impact on contrast induced acute kidney injury. J Cardiol 63: 9-13.

49. Ogasawara S, Mukawa H, Sone T, Tsuboi H, Morishima I, et al. (2015) Presence of myocardial hypoenhancement on multidetector computed tomography after primary percutaneous coronary intervention in acute myocardial infarction predicts poor prognosis. Int J Cardiol 184: 101-107.

50. Christopoulos G, Karmpaliotis D, Wyman MR, Alaswad K, McCabe J, et al (2014) Percutaneous intervention of circumflex chronic total occlusions is associated with worse procedural outcomes: insights from a Multicentre US Registry. Can J Cardiol 30: 1588-1594.

51. Brown JR, Solomon RJ, Sarnak MJ, McCullough PA, Splaine ME, et al. (2014) Reducing contrast-induced acute kidney injury using a regional multicenter 
Citation: Ladwig M, Cantow K, Flemming B, Persson PB, Seeliger E. Comparison of the Effects of lodixanol and lopamidol on Urine Flow, Urine Viscosity, and Glomerular Filtration in Rats. J Urol Nephrol. 2015;2(1): 7.

ISSN: $2380-0585$

quality improvement intervention. Circ Cardiovasc Qual Outcomes 7: 693 700

52. Liu W, Wagatsuma K, Toda M, Amano H, Nii H (2011) Short- and long-term follow-up of percutaneous coronary intervention for chronic total occlusion through transradial approach: tips for successful procedure from a singlecenter experience. J Interv Cardiol 24: 137-143.

53. U.S. Department of Health and Human Services, Food and Drug
Administration, Center for Drug Evaluation and Research (2005) Guidance for Industry: Estimating the maximum safe starting dose in initial clinical trials for therapeutics in adult healthy volunteers.

54. Barrett BJ, Katzberg RW, Thomsen HS, Chen N, Sahani D, et al. (2006) Contrast-induced nephropathy in patients with chronic kidney disease undergoing computed tomography: a double-blind comparison of iodixanol and iopamidol. Invest Radiol 41: 815-821. 